\title{
Atmospheric Profiling in the Inter-Tropical Ocean Area Based on Neural Network Approach Using GPS Radio Occultations
}

\author{
Fabrizio Pelliccia ${ }^{1}$, Stefania Bonafoni ${ }^{*},{ }^{1}$, Patrizia Basili ${ }^{1}$, Piero Ciotti ${ }^{2}$ and Nazzareno Pierdicca ${ }^{3}$ \\ Università degli Studi di Perugia, Dipartimento di Ingegneria Elettronica e dell'Informazione, via G. Duranti,93 - \\ 06125 Perugia, Italy \\ Università di L'Aquila, Dipartimento di Ingegneria Elettrica e dell'Informazione, Poggio di Roio - 67040 L'Aquila, \\ Italy \\ Università di Roma "La Sapienza”, Dipartimento di Ingegneria Elettronica, via Eudossiana, 18 - 00184 Roma, Italy
}

\begin{abstract}
In this study we have proposed a method based on neural networks to retrieve refractivity, temperature, pressure and humidity profiles by using FORMOSAT-3/COSMIC GPS radio occultation data. To overcome the constraint of an independent knowledge of one atmospheric parameter at each GPS occultation, we trained three neural networks with refractivity profiles as input computed from the geometrical occultation parameters relative to the FORMOSAT3/COSMIC satellites, while the targets were the dry and wet refractivity profiles and the dry pressure profiles obtained from the contemporary European Centre for Medium-Range Weather Forecast data. We have considered 1041 available satellite radio occultations covering the entire ocean area spanning within the Tropics during July-August 2006. We have used 937 profiles for training the neural networks, the remaining 104 ones for the independent test.
\end{abstract}

Keywords: GPS, radio occultation, neural network, atmospheric profiling.

\section{INTRODUCTION}

Global Positioning System (GPS) radio occultation (RO) is considered as a global sounding technique for providing atmospheric profiles useful for numerical weather prediction and climate change studies. The radio occultation system employs GPS receivers placed on Low-Earth Orbit (LEO) satellites to sound the Earth's neutral atmosphere and ionosphere evaluating the additional delay affecting a radio signal when passing through the atmosphere due to the refractivity index magnitude and its variations $[1,2]$. Since 1995, when the first LEO satellite Microlab-1 (GPS/MET mission) was operational $[3,4]$, several GPS-RO satellite missions have been launched, which include Stellenbosch University Satellite (SUNSAT), Challenging Mini-Satellite Payload (CHAMP), Satelite de Aplicaciones Cientificas-C (SAC-C), Gravity Recovery and Climate Experiment (GRACE) and Constellation Observing System for Meteorology Ionosphere and Climate (COSMIC).

GPS occultations provide an active probing of the atmosphere working under all-weather conditions due to the insensitivity of the GPS signal wavelength to scattering by clouds, aerosols, and precipitation, with relatively high vertical resolution throughout the depth of the atmosphere associated with the limb-viewing geometry. A limiting factor for this technique is the horizontal resolution. It is set by the Fresnel diffraction-limited pencil-shaped sampling volume

*Address correspondence to this author at the Università degli Studi di Perugia, Dipartimento di Ingegneria Elettronica e dell'Informazione, via G. Duranti, 93 - 06125 Perugia, Italy; Tel: $+39-75 / 585$ 3663; Fax: $+39-75 / 585$ 3654; E-mail: stefania.bonafoni@diei.unipg.it of each measurement which has a horizontal resolution of about $200 \mathrm{~km}$ in the direction along the occulted link and a resolution of $1 \mathrm{~km}$ or better in the cross-link and vertical directions [5].

The GPS-RO technique is exploited to obtain profiles of refractivity, temperature, pressure and humidity in the atmosphere at global scale, and several investigations have demonstrated that the retrieval accuracies are comparable to traditional atmospheric remote sensing techniques $[6,7]$. Even though the atmospheric refractivity profiling by radio occultation is a well-defined problem, care must be taken to analyze factors affecting the occulted signal (multipath, satellite motion etc.) and to compute temperature and particularly humidity profiles from refractivity [5]. The accuracy of atmospheric profile estimation is affected by the use of proper boundary conditions and by the presence of water vapour in the atmosphere, that complicates the interpretation of the refractivity [8]. Refractivity profiles can be converted in a straightforward way into pressure and temperature profiles in regions where water vapour is negligible such as in the upper troposphere.

In the middle and lower troposphere additional information is necessary, therefore GPS refractivity measurements are employed to derive profiles of water vapour partial pressure or specific humidity given an independent knowledge of temperature obtained from independent observations (i.e. radiosoundings or data from atmospheric numerical modeling).

For example, an iterative method exploiting GPS RO refractivity measurements and temperature profiles obtained from radiosoundings or models to derive profiles of water 
vapor partial pressure and specific humidity is discussed in [1]. An improved and widely used approach for atmospheric profiling, known as one-dimensional variational assimilation (1D-var) [9], consists in solving for the optimal onedimensional atmospheric state, i.e. optimal pressure, temperature and water vapor partial pressure, using observed GPS RO refractivity and a background atmospheric state from a model, each accompanied by its own error characterization.

The main drawbacks of these methods consist essentially in their dependence on additional sources of information at each GPS occultation, and the need of post-processing RO data to obtain the target atmospheric profiles.

In this paper, a retrieval method based on neural networks is proposed to retrieve profiles of atmospheric parameters from $\mathrm{RO}$ refractivity overcoming the requirement for temperature profile availability at each GPS occultation. We have trained three neural networks with inputs consisting of refractivity profiles computed from the occultation parameters observed by the COSMIC Microsat Constellation satellites and provided by the COSMIC Data Analysis and Archive Center (CDAAC) of Boulder (Colorado) [10]. The targets employed in the training are the dry and wet refractivity profiles, together with the dry pressure ones, obtained from the contemporary European Centre for Medium-Range Weather Forecast (ECMWF) analysis data.

ECMWF data are used only in the training phase of the neural networks. Once trained, they are capable of providing in real time the decomposition into wet and dry components of the incoming RO refractivity, thus allowing one to obtain temperature and water vapor pressure profiles, without requiring independent data sets.

The neural network training and the following independent test were performed over the entire ocean area between Tropics by using the available data set of 1041 refractivity profiles from July 17 to August 18 2006. We chose to split ocean from land RO events: in a complementary paper [11] the performances of four $\mathrm{NN}$ algorithms are compared considering the same dataset of RO events selected over land.

The output decomposition in wet and dry refractivity and the estimation of dry pressure allows to obtain temperature and water vapour pressure profiles, without requiring independent information on atmospheric temperature.

To evaluate the performances of the proposed approach in processing $\mathrm{RO}$ data and to assess the quality of the retrieved profiles, we have computed the errors affecting the estimated profiles (refractivity, temperature, dry and wet pressure) with respect to ECMWF analysis which have been assumed as the truth or anyway as a good approximation of the real atmosphere. Such a choice of ECMWF data as a reference in the comparison was also adopted by other authors [12-14], considering that these data provide global coverage and high spatial resolution reconstruction of the global atmosphere. Of course, the use of ECMWF analysis occasionally might wrongly correlate atmospheric variables: a true validation with independent and actual measurements, such as radiosoundings (RAOBs), would provide a better benchmark, but over sea RAOBs are not available.
Our paper is structured as follows: at first, we explain the method used to extrapolate refractivity profiles from geometrical parameters of RO and atmospheric parameters from the acquired ECMWF data; then we deal with the chosen methodology to retrieve atmospheric profiles overcoming the need of knowing the temperature profile at each GPS occultation; finally we describe the neural network approach and we assess the quality of the obtained profiles with respect to ECMWF analysis ones.

\section{ATMOSPHERIC PROFILE RETRIEVAL}

\subsection{Atmospheric Bending and Refractivity Profiles Retrieval}

GPS-RO observations are performed in a limb-scanning mode, where in the geometrical optics approximation a ray passing through the atmosphere is refracted due to the vertical refractive profile. The overall effect of the atmosphere can be characterized by a total bending angle $\alpha$, an asymptotic impact parameter $a$ and a tangent radius $r_{p}$, as shown in Fig. (1), and their variations depend primarily on the vertical profile of refractive index [5].

With the assumption of local spherical symmetry, the refraction index profile $n$ can be retrieved from measurements of $\alpha$ as a function of $a$ during an occultation by using an Abel transformation as in [15]:

$n\left(r_{p}\right)=\exp \left(\frac{1}{\pi} \int_{a_{r_{p}}}^{\infty} \frac{\alpha(a)}{\sqrt{a^{2}-a_{r_{p}}^{2}}} d a\right)$

where $a_{r p}=n\left(r_{p}\right) \cdot r_{p}$ is the impact parameter for the ray whose tangent radius is $r_{p}$. The refractivity profile is then $N=(n-$ $1) \cdot 10^{6}$. Also, the "occultation point" is defined as the point on the Earth's surface to which the retrieved refractivity profile is assigned, located under the perigee point of the bended ray [13].

\subsection{Selection of Radio Occultation Data}

In this work, first we have collected all the 1718 FORMOSAT-3/COSMIC radio occultation events provided by CDAAC, covering the inter-tropical ocean area during the period from July 17 to August 18, 2006. The FORMOSAT$3 /$ COSMIC is a joint Taiwan - U.S. mission that provides a constellation of six identical micro-satellites successfully launched on April 14, 2006. The receivers installed onboard of the satellites register the phase and the amplitude of radio waves at the two GPS carrier frequencies, L1 (1575.42 $\mathrm{MHz})$ and L2 (1227.6 MHz), useful for the computation of the geometrical parameters introduced in section 2.1.

Then, we have computed the refractivity profiles using the geometrical parameters as in (1). The impact parameters and the bending angles employed in this study are contained in the atmPrf product collected in the level 2 atmospheric profile section of the CDAAC, vertically spaced from $4 \mathrm{~m}$ to $50 \mathrm{~m}$ in the low and high atmosphere, respectively.

The distribution of the considered GPS-RO events is shown in Fig. (2), by marking the ones used for the neural network training with stars and those for the network test with open circles. 


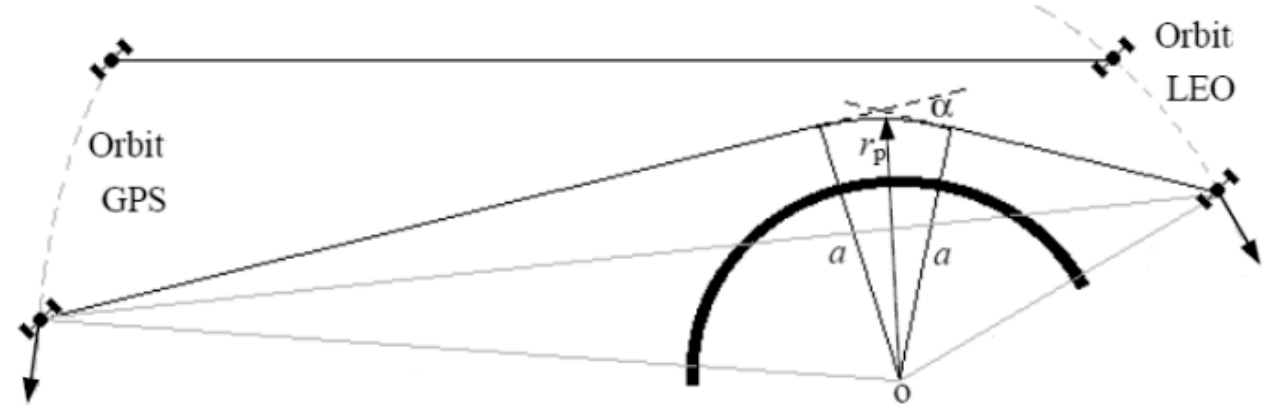

Fig. (1). Instantaneous GPS-LEO occultation parameters.

In this work, we have produced a complete database matching the FORMOSAT-3/COSMIC GPS occultations with the corresponding ECMWF observations co-located in space and time. The RO profiles and the ECMWF data have been co-located on the basis of the correspondence between the terrestrial coordinates of the occultation points of $\mathrm{RO}$ and those provided in ECMWF data, with a maximum geographical coordinate distance of $0.5^{\circ}$ and a maximum time difference of 1 hour. Data regarding only ocean area have been chosen on the basis of the land/sea flag included in the ECMWF data.

A possible source of errors in the occultations is a large difference between L1 and L2 bending angles, that could indicate extreme ionospheric conditions or L2 tracking errors. Another source of errors is a large absolute value of the mean deviation smean of the observed ionosphere free bending angle from CIRA-86 (COSPAR International Reference Atmosphere) climatological model, between 60 and $80 \mathrm{~km}$. A great value of smean could invalidate the occultation event above the altitude of $20 \mathrm{~km}$. Although in this work we have considered only altitudes under $20 \mathrm{~km}$, because usually above this altitude the observed bending angle profiles are weighted with the first guess bending angles derived from the CIRA-86 model [13], we have carefully rejected all the occultations (19 events from the above-mentioned collection) presenting an absolute value of smean greater than $10^{-4} \mathrm{rad}$.
In order to train and test the neural networks we have created a database of refractivity profiles by interpolating all parameters involved in the computation of refractivity at the same altitude intervals at each observation. As a result, each profile has 689 fixed altitude levels, representing the atmosphere from 0.9 to $20 \mathrm{~km}$.

By analyzing the remaining 1699 events, we have chosen the altitude of $0.9 \mathrm{~km}$ as the minimum height above the surface as a trade-off between the requirement of a great number of observations to train the networks and the need of atmospheric profile estimation as much as possible close to the surface. On the other hand, such a choice limits the knowledge of the atmosphere at lower levels where substantial deviations of normal refractivity gradients may occur, typically due to super-refractive or, sometimes, ducting layers $[16,17]$.

Therefore, discarding RO ending at an altitude higher than $0.9 \mathrm{~km}$, we have finally selected 1041 occultations, and hence 1041 impact parameter and bending angle profiles to compute refractivity.

\subsection{Conventional Retrieval of Atmospheric Profiles}

The atmospheric refractivity at microwave wavelength is given by [18]:

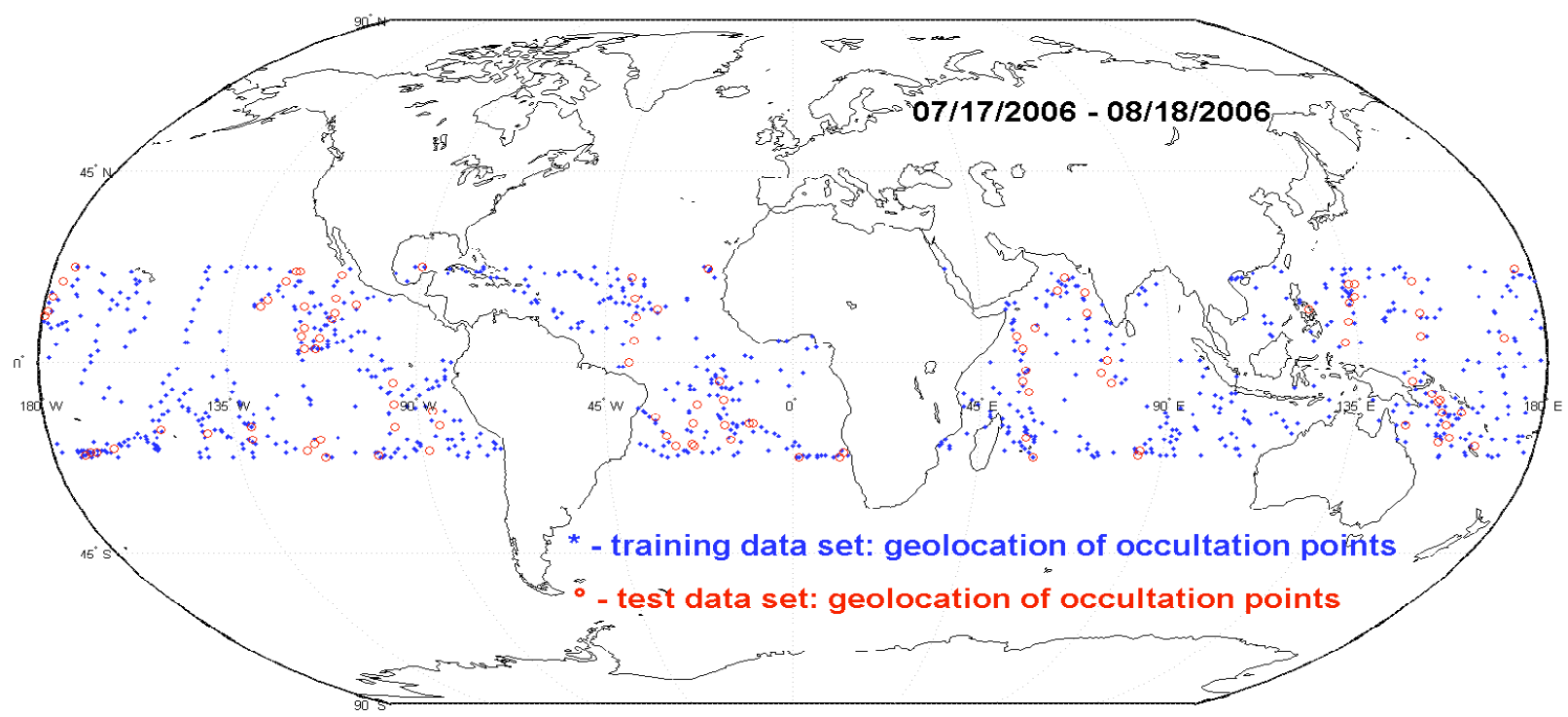

Fig. (2). Distribution of FORMOSAT-3/COSMIC GPS occultations. 
$N=77.6 \frac{P_{d}}{T}+72 \frac{P_{w}}{T}+3.75 \cdot 10^{5} \frac{P_{w}}{T^{2}}$

where $P_{d}$ is the pressure of dry air in $\mathrm{hPa}, P_{w}$ the partial pressure of water vapour in $\mathrm{hPa}, T$ is the atmospheric temperature in Kelvin. This equation is accurate to within $0.5 \%$ for frequencies up to $100 \mathrm{GHz}$. To solve for $T, P_{d}$ and $P_{w}$ given $N$, we use the additional constraints of ideal gas and hydrostatic equilibrium laws, respectively, as:

$\rho=\frac{P}{T} \frac{M_{d}}{R_{0}}+\frac{P_{w}}{T} \frac{\left(M_{w}-M_{d}\right)}{R_{0}}$

$d P(z)=-g \rho(z) d z$

where $\rho(z)$ is the air density in $\mathrm{kg} \mathrm{m}^{-3}, P=P_{d}+P_{w}, M_{d}$ and $M_{w}$ are respectively the mean molecular mass of dry air and water vapour, $R_{0}$ is the universal gas constant, $g$ the gravitation acceleration. Given $N$, we have a system of three equations and four unknowns $\left(T, P_{d}, P_{w}\right.$ and $\left.\rho\right)$, and therefore it is necessary to have an independent knowledge of one of the four parameters to solve the atmospheric profiling problem [12, 19]. For instance, some authors exploit the temperature profile derived from independent observations or weather analysis [5]. In this study, a method based on neural networks is proposed to retrieve atmospheric profiles overcoming the need of knowing the temperature profile at each GPS occultation.

\subsection{Refractivity and Pressure from ECMWF Data}

The targets for the neural network training and the references for the following test are the dry and wet refractivity profiles and the dry pressure profiles. These profiles were obtained by processing data provided by the ECWMF, such as the logarithm of pressure, the specific humidity and the temperature, belonging to the "ECMWF 91 model levels" data set, representing the atmosphere from 75 $\mathrm{km}$ to the ground with a vertical resolution spanning from 5 $\mathrm{km}$ to $25 \mathrm{~m}$ in the high and low atmosphere, respectively.

From ECMWF analysis data we have computed the water vapour partial pressure profile $P_{w}$ by using the relationship between $P_{w}$ and mixing ratio:

$$
P_{w}=\frac{w P}{\frac{R_{d r y}}{R_{\text {vap }}}+w}
$$

where $w$ is the mixing ratio in $\mathrm{kg} \mathrm{kg}^{-1}$ derived from the specific humidity, $R_{d r y}$ and $R_{\text {vap }}$ are the gas constant for dry air and water vapour respectively and $P$ is the total pressure in $\mathrm{hPa}$. Hence, the dry pressure profile has been obtained by subtracting the partial pressure of water vapour from the total pressure profile. Finally we have computed the refractivity by using (2) and than we have vertically interpolated these profiles at the same vertical levels of the refractivity profiles obtained from Abel transformation, as described in section 2.2.

\section{NEURAL NETWORK APPROACH}

To solve the atmospheric profiling problem of GPS LEO occultations overcoming the need of external information (i.e., temperature profiles), we have considered three neural networks where predictors are the total refractivity profiles $N(z)$ provided by the RO technique using (1) and the targets are the corresponding dry $N_{d}(z)$ and wet $N_{w}(z)$ refractivity profiles and the dry pressure profiles $P_{d}(z)$ computed from ECMWF data. $N_{d}(z)$ is the first term on the right-hand side of (2), while $N_{w}(z)$ is the second and third term. Note that the consideration of ECMWF data allow the neural network to reproduce similar relations between the variables as those found in the training data set, and thus producing profiles coherent with those physical constraints.

The neural network training and the following independent test were performed over the entire ocean area between Tropics by using the available data set of 1041 refractivity profiles, representing the atmosphere from 0.9 to $20 \mathrm{~km}$. We have chosen, randomly, 937 profiles for the training and the remaining 104 for the independent test of the network, that represent $90 \%$ and $10 \%$ of the entire available dataset, respectively.

First, before the training session, we have processed input and target matrices, with columns representing atmospheric parameter profiles, standardizing each row's means to 0 and standard deviations to 1 [20]. After standardization, we have processed the input and target matrices using Principal Component Analysis (PCA) by expanding the 689-level refractivity profiles on a basis of empirical orthogonal functions called principal components [21]. The PCA permits a reduction of the number of descriptive profile parameters by exploiting the correlation among values at different altitudes, ensuring a faster postprocessing and a reduction of computer memory requirements in comparison with the original data. We have chosen to employ a number of principal components representing the $99.9 \%$ of the total variance of the original data [20], leading to the use of only 22 principal components for the total refractivity instead of the original 689 levels. Concerning the neural network targets, the number of components for dry refractivity, wet refractivity and dry pressure profiles are 17, 20 and 10, respectively. The need of a bigger number of wet refractivity principal components with respect to dry refractivity and dry pressure is an evidence of a greater variability in the troposphere of the wet parameters with respect to the dry ones.

For the training session of the neural networks, we have applied the early stopping technique, useful for determining the optimal number of training epochs. The early stopping technique divides the available events in two disjoint subsets: the training set and the validation set. The first one is used for the learning itself, the second one to choose the number of training epochs. Learning ends when the error on the validation set begins to rise even if the error on the training set could be further reduced. In practice, the validation set improves the ability of generalization of the network. Since overtraining could occur even on the validation set, a further test subset should be used to assess the capacity of generalization of the network. Then we have divided the training data set (937 events) in three subsets: the training subset used for the learning itself, the validation subset and the test subset, by assigning them randomly the $70 \%$ (655 events), the 15\% (141 events) and the 15\% (141 events) of the whole data set, respectively. 
Instead of the standard back-propagation, we have used the Levenberg-Marquardt optimization that is often the fastest back-propagation algorithm for training moderatesized feed-forward neural networks, in agreement with the early stopping technique $[22,23]$. We have considered feedforward neural networks having, besides the input layer, a number (1 to 3 ) of hidden layers with tan-sigmoid transfer functions and an output layer with linear transfer functions. The degrees of freedom in designing the neural network architecture are two: the number of hidden layers and the number of neurons per layer. To select the more suitable architecture, we have added 1 neuron starting from the first hidden layer at each training session, until a maximum of 20 neurons for each hidden layer. We have considered a maximum of 3 hidden layers, choosing among the possible combinations the architecture with the lower root mean square (RMS) error computed comparing the network outputs of the test session with the corresponding ECMWF profiles, where the test session employs the 104 refractivity profiles not used in the training phase. The best performance for the dry refractivity retrieval has been obtained using a neural network with 1 hidden layer with 8 neurons. Also, the neural network for the wet refractivity includes 1 hidden layer of 10 neurons, while the one for dry pressure includes 1 hidden layer of 5 neurons.

\section{RESULTS}

The integration of (1) introduces some systematic errors on the estimation of the total refractivity profiles $N$. The hypothesis of a spherically symmetrical atmosphere, implied in the Abel transform inversion, produces a small error due to the ellipsoidal shape of the Earth and to the horizontal gradients of the atmospheric structure [24, 25]. RO data analysis centers, as CDAAC, mitigate the error associated to the hypothesis of spherical symmetry by selecting Earth center and radius of curvature appropriate to the latitude and orientation of occultation measurements [25]. Another approximation is the assumption of the co-planarity of the ray paths during an entire occultation [5]. For these reasons, taking into account the low horizontal resolution of RO and the possible errors of the ECMWF data, the comparison between the total refractivity profiles $N$ obtained using Abel transformation and the corresponding ECMWF $N$ profiles exhibits a not negligible root mean square (RMS) difference, that the network anyway contributes slightly to reduce, as a sort of calibration, as reported in Fig. (3). The RMS error profile of $N$ from Abel transformation (green line) is shown in Fig. (3) superimposed to the corresponding ECMWF standard deviation profile (red line). The latter defines the standard deviation profile of the atmospheric parameter computed from the entire ECMWF database: it can be assumed as an index of the climatological variability of a given parameter, than a good accuracy of a retrieval algorithm is obtained when its RMS error is clearly below it. Also, the RMS error profile computed from the comparison between $N$ obtained as output of the neural network training $\left(N=N_{d}+N_{w}\right)$, i.e. the autotest result, and the corresponding ECMWF profiles is shown in Fig. (3) (blue line), exhibiting a vertically averaged RMS error of 2.78 (N unit). The vertically averaged RMS error of $N$ from Abel transformation is 3.58 ( $\mathrm{N}$ unit), while the mean standard deviation of the entire ECMWF database is 6.13 ( $\mathrm{N}$ unit).

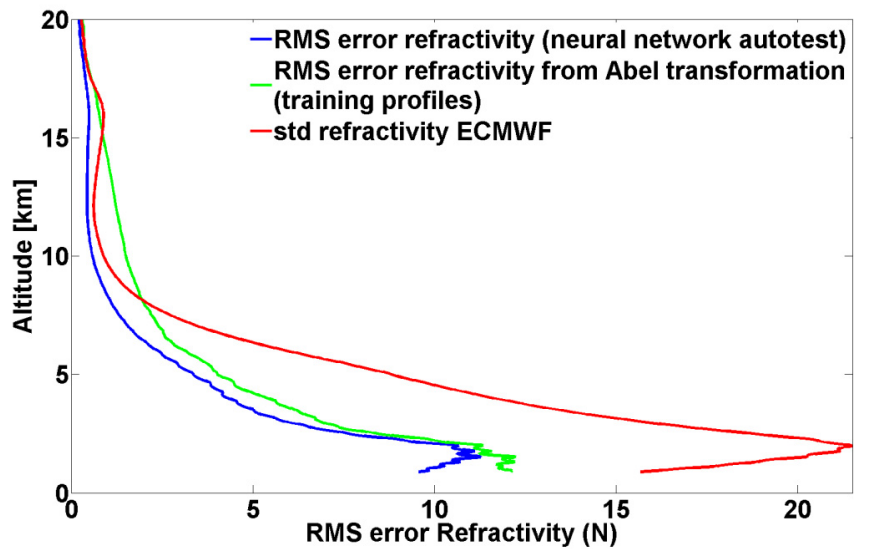

Fig. (3). RMS error profile for $N$ (blue line) obtained as output of the neural network training (autotest, 937 occultations), RMS error profile for $N$ from Abel transformation (green line) using the same 937 occultations and ECMWF standard deviation profile (red line).

To evaluate the performances of the neural networks on the ability to reconstruct $N_{d}, N_{w}$ and $P_{d}$ profiles from $N$ obtained from the Abel transform, the RMS error profiles for $N_{d}, N_{w}$ and $P_{d}$ (green line) employing the independent test set of 104 occultations are shown in Figs. (4-6), respectively, superimposed to the corresponding ECMWF standard deviation profiles (red line). For $N_{d}$, the vertically averaged RMS error is 0.76 ( $\mathrm{N}$ unit), while the corresponding mean standard deviation of the entire ECMWF database is $1.21(\mathrm{~N}$ unit). For $N_{w}$, the vertically averaged RMS error is $2.73(\mathrm{~N}$ unit), while the mean standard deviation of the corresponding ECMWF database is 6.34 ( $\mathrm{N}$ unit). Finally, for $P_{d}$, the vertically averaged RMS error is $1.61 \mathrm{hPa}$ against a mean standard deviation ECMWF of $2.55 \mathrm{hPa}$. In particular, the use of the neural networks tend to cut down the bias error.

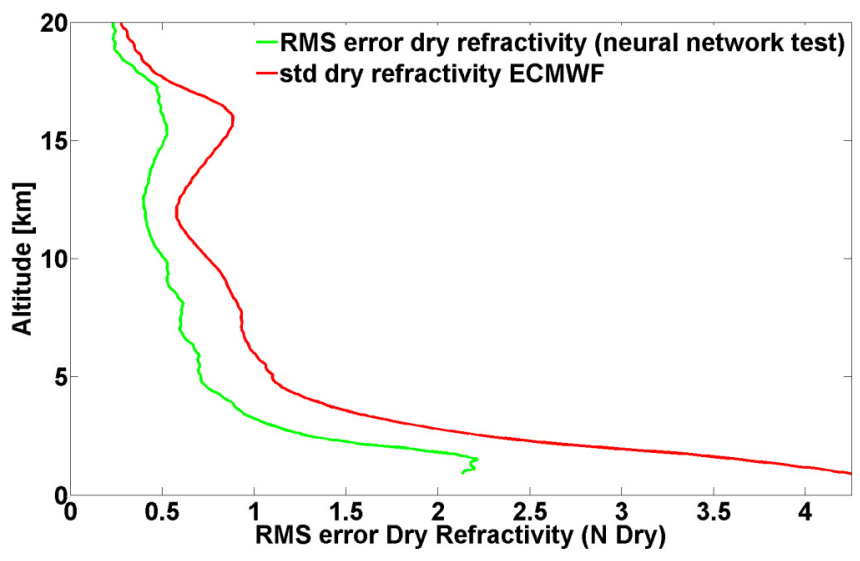

Fig. (4). Neural network independent test (104 occultations): profile of RMS error for $N_{d}$ (green line) and ECMWF standard deviation profile (red line).

The performances of the trained networks have been evaluated by performing a regression analysis between the network responses and the corresponding targets data [20]. The regression plots for $N_{d}, N_{w}$ and $P_{d}$ are shown in Figs. (79), respectively. For each parameter, the network outputs are plotted versus the targets as open circles, the best linear regression fit is indicated by a continuous line and the perfect fit, that relate outputs equal to targets, is indicated by a dashed line. Concerning the dry parameters, the best linear 
fits relating targets to network outputs are very close to the corresponding perfects fits, by showing respectively for $N_{d}$ and $P_{d}$ slope values equal to 1 and y-intercept values equal to 0.08 and -0.15 . Also, the dry parameters $N_{d}$ and $P_{d}$ exhibit a quasi-perfect correlation between targets and outputs by presenting values of correlation coefficients (R-values) equal to 0.999 for both. Wet refractivity $N_{w}$ profiles show also a good R-value of 0.964 , but the tendency of the best linear fit is worse with respect to those of dry parameters (slope of 0.93 and $y$-intercept of 0.74), giving evidence of the complex behavior of the wet components due to their greater variability compared to the dry parameters.

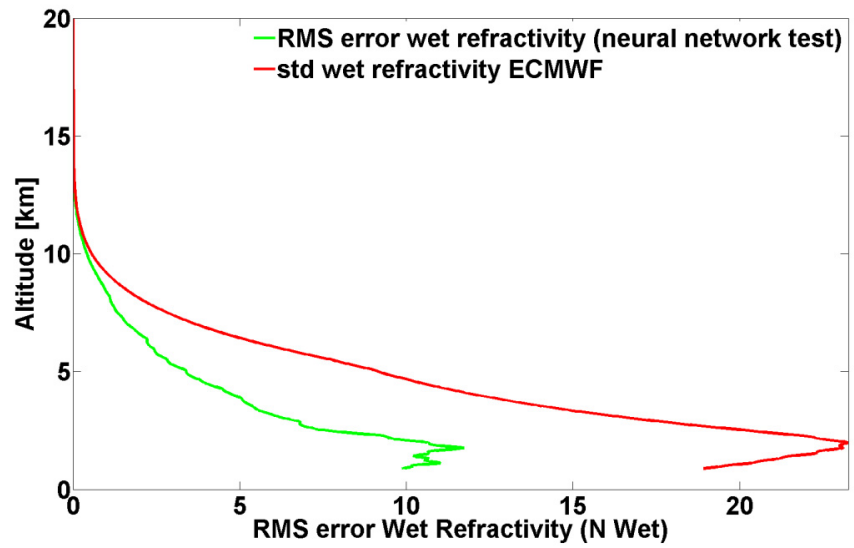

Fig. (5). Neural network independent test (104 occultations): profile of RMS error for $N_{w}$ (green line) and ECMWF standard deviation profile (red line).

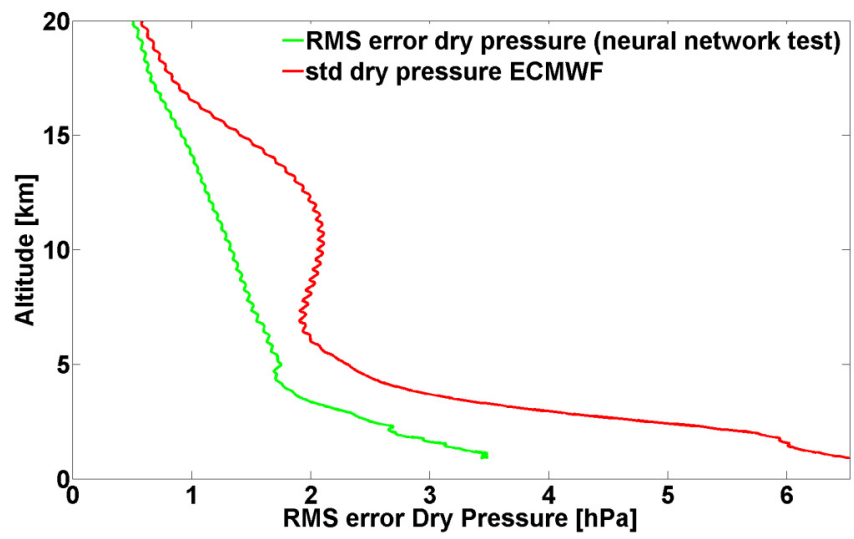

Fig. (6). Neural network independent test (104 occultations): profile of RMS error for $P_{d}$ (green line) and ECMWF standard deviation profile (red line).

Furthermore, to evaluate the ability of generalization of the neural networks, the RMS error profile for the total refractivity $N$ estimated in the test session $\left(N=N_{d}+N_{w}\right.$, green line) and the RMS error profile for $N$ estimated in the training session, or autotest (blue line), are shown in Fig. (10), superimposed to the ECMWF standard deviation profile (red line). The two RMS error profiles exhibit an evident correspondence, pointing out the neural network ability to identify the correct refractivity profiles despite the independent data set they belong to.

Also, we have chosen to estimate the dry pressure $P_{d}$ from the network instead of solving the ideal gas and hydrostatic equilibrium laws in dry conditions, since the error introduced by the neural network is significantly lower than the one obtained by the numerical integration through the trapezium rule of (4) after using (2) and (3) in dry conditions. Of course, the dry pressure employed in the neural network learning, computed from ECMWF analysis data, obeys the hydrostatic equation.

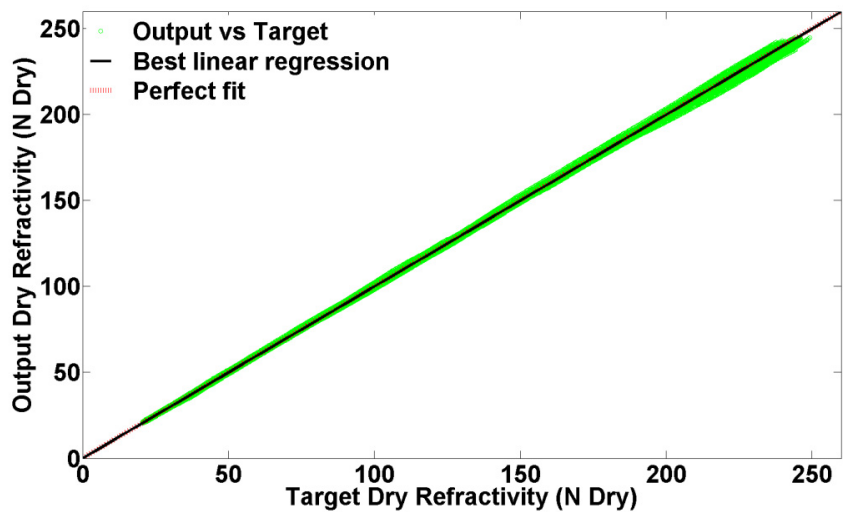

Fig. (7). Neural network independent test (104 occultations) for $N_{d}$ : output versus target (open green circles), best linear regression fit (black line), perfect fit (dashed red line).

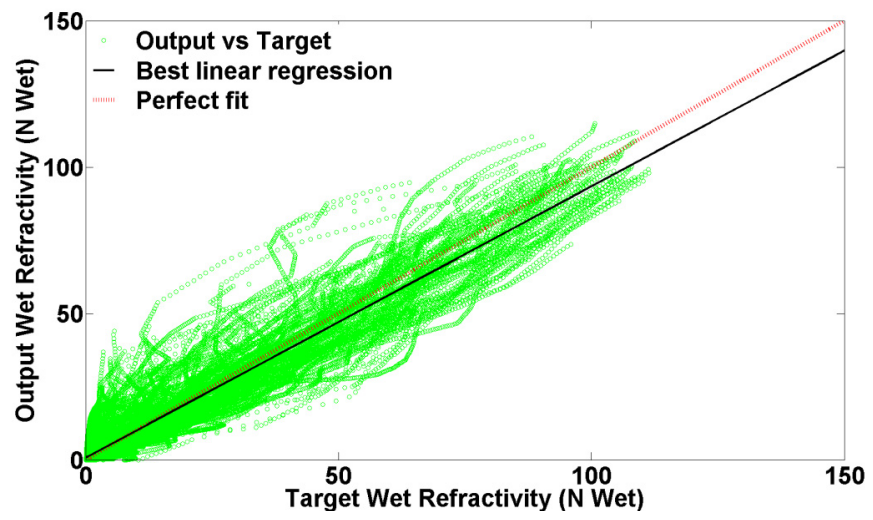

Fig. (8). Neural network independent test (104 occultations) for $N_{w}$ : output versus target (open green circles), best linear regression fit (black line), perfect fit (dashed red line).

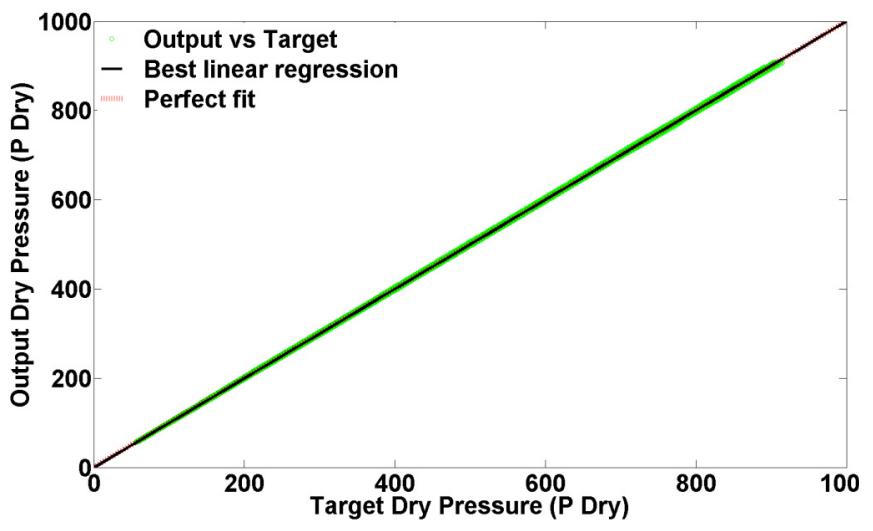

Fig. (9). Neural network independent test (104 occultations) for $P_{d}$ : output versus target (open green circles), best linear regression fit (black line), perfect fit (dashed red line).

The choice to train three networks for the three outputs is justified by the necessity of retrieving atmospheric profiles without the constraint of temperature profile availability at each GPS occultation, as required to solve the system of (2), (3), (4). With the availability of $N_{d}, N_{w}$ and $P_{d}$, first we can 
solve for temperature $T$ in a straightforward way from the dry refractivity relation

$N_{d}=77.6 \frac{P_{d}}{T}$

and then for partial pressure of water vapor $P_{w}$ from the wet refractivity relation

$N_{w}=72 \frac{P_{w}}{T}+3.75 \cdot 10^{5} \frac{P_{w}}{T^{2}}$

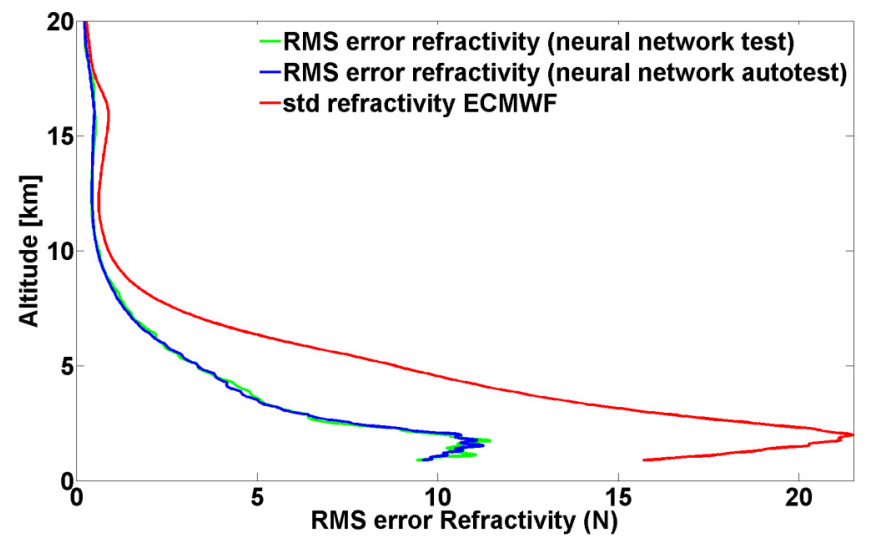

Fig. (10). RMS error profiles for $N$ from neural network test (green line) and from neural network autotest (blue line) and ECMWF standard deviation profile (red line).

In Figs. $(11,12)$ the RMS error profiles for $T$ and $P_{w}$ (green line) are shown superimposed to the corresponding ECMWF standard deviation profiles (red line).

For $T$, the vertically averaged RMS error is $1.53 \mathrm{~K}$ while the corresponding mean standard deviation of ECMWF database is $2.22 \mathrm{~K}$. For $P_{w}$, the vertically averaged RMS error is $0.54 \mathrm{hPa}$ against a mean standard deviation ECMWF of $1.27 \mathrm{hPa}$.

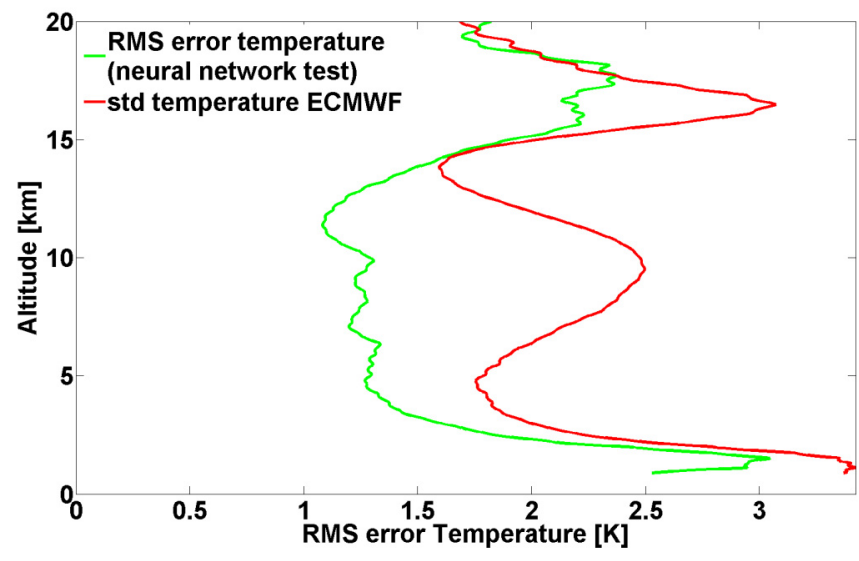

Fig. (11). Neural network independent test (104 occultations): RMS error profile for $T$ (green line) and ECMWF standard deviation profile (red line).

\section{CONCLUSIONS}

In this work, we have proposed a method to estimate profiles of refractivity, temperature, pressure of dry air and of water vapour in the troposphere from FORMOSAT3/COSMIC GPS radio occultation over the entire ocean area between Tropics. The work has regarded July-August climatic conditions.

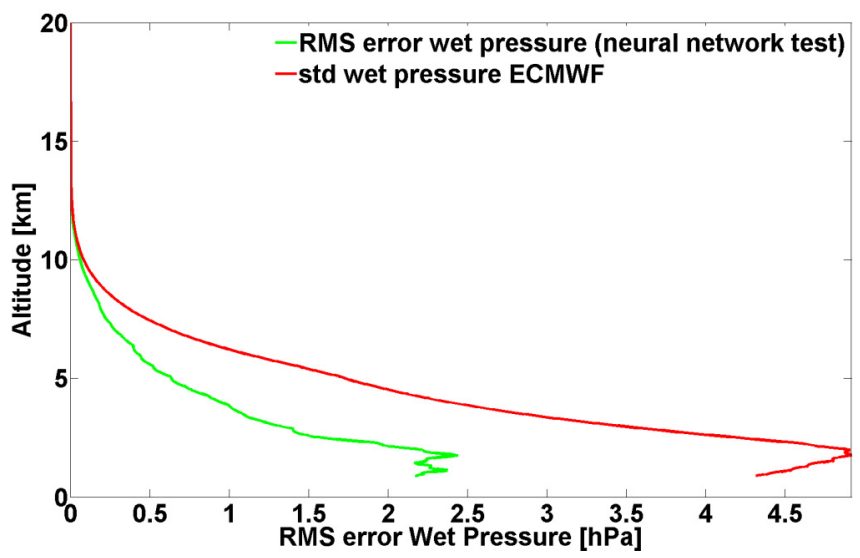

Fig. (12). Neural network independent test (104 occultations): RMS error profile for $P_{w}$ (green line) and ECMWF standard deviation profile (red line).

To overcome the necessity to know the true temperature profile at each occultation, we have trained three neural networks with targets that permit to solve the atmospheric profiling problem by using only (2), and to reflect the physical constraints learned from the ECMWF analysis data.

The results have shown good performances of the neural networks using the principal component analysis for a fast and less expensive approach, exhibiting a fairly good accuracy for temperature and partial pressure of water vapour profiles. However, wet parameter retrieval exhibits lower accuracies than dry one, due to their complex behavior and the greater variability of the wet components of the troposphere.

Furthermore, such results can be improved with the future availability of a larger data set for training the neural network, covering a wider variety of atmospheric conditions spanning a larger seasonal and geographical extension.

In conclusion, our analysis sets itself the goal of showing the possibility to retrieve each atmospheric parameter included the wet ones only from RO refractivity, and then the ability to increase the atmospheric observations, integrating them successively in the NWP models, thanks to a wide spatial coverage of RO soundings on the Earth (only COSMIC mission produces 1500-2500 profiles per day). The bound of this approach is that the informative contribution brought by RO soundings is in some way connected to the necessary employment of the ECMWF atmospheric model profiles as targets for the neural network training.

\section{ACKNOWLEDGEMENTS}

The work has been sponsored by the ASI, Italian Space Agency, through the Thales Alenia Space. We wish to thank COSMIC Data Analysis and Archive Center (CDAAC) of Boulder (Colorado) for the availability of the occultation data.

\section{REFERENCES}

[1] Gorbunov ME, Sokolovskiy SV. Remote sensing of refractivity from space for global observations of atmospheric parameters. 
Max-Planck-Institut fur Meteorologie, Hamburg, 1993; Report No. 119.

[2] Rius A, Ruffini G, Romeo A. Analysis of ionospheric electron density distribution from GPS/MET occultations. IEEE Trans Geosci Remote Sensing 1998; 36(2): 383-94.

[3] Ware R, Exner M, Feng D, et al. GPS Sounding of the atmosphere from low Earth orbit: preliminary results. Bull Am Meteorol Soc 1996; 77: 19-40

[4] Rocken C, Anthes R, Exner, et al. Analysis and validation of GPS/MET data in the neutral atmosphere. J Geophys Res 1997; 102(D25): 29849-66.

[5] Kursinski ER, Hajj GA, Schofield JT, Linfield RP, Hardy KR. Observing Earth's atmosphere with radio occultation measurements using the Global Positioning System. J Geophys Res 1997; 102(D19): 429-65

[6] Hardy KR, Hajj GA, Kusinski ER. Accuracies of atmospheric profiles obtained from GPS occultation. Int J Satell Comm 1994; 12: 463-73.

[7] Kursinski ER, Hajj GA, Hardy KR, Romans LJ, Schofield JT. Observing tropospheric water vapor by radio occultation using the Global Positioning system. Geophys Res Lett 1995; 22: 2365-8.

[8] O'Sullivan DB, Hermann BM, Feng D, Flittern DE, Ward DM. Retrieval of Water Vapor Profiles from GPS/MET Radio Occultations. Bull Am Metereol Soc 2000; 81(5): 1031-40.

[9] Kuo YH, Sokolovskiy S, Anthes RA, Vandenberghe F. Assimilation of GPS radio occultation data for numerical weather prediction. Special issue of Terrestrial, Atmospheric and Oceanic Sci 2000; 11(1): 157-86.

[10] Anthes RA, Rocken C, Kuo YH. Applications of COSMIC to meteorology and climate. Terr Atmos Ocean Sci 2000; 11(1): 115 56.

[11] Bonafoni S, Pelliccia F, Anniballe R. Comparison of different neural network approaches for the tropospheric profiling over the inter-tropical lands using GPS radio occultation data. Algorithms 2009; 2: 31-45.

[12] Kursinski ER, Hajj GA. A comparison of water vapour derived from GPS occultations and global weather analyses. J Geophys Res 2001; 106(D1): 1113-38.
[13] Kuo Y H, Wee T K, Sokolovskiy S, Rocken C, Schreiner C, Hunt $\mathrm{D}$, Anthes RA. Inversion and error estimation of GPS Radio Occultation data. J Meteorol Soci Jap 2004; 82(1B): 507-31.

[14] Beyerle G, Schmidt T, Wickert J, Heise S, Rothacher M, KonigLanglo $\mathrm{G}$, Lauritsen $\mathrm{KB}$. Observations and simulations of receiverinduced refractivity biases in GPS radio occultation. J Geophys Res 2006; 111(D12).

[15] Fjeldbo G, Kliore AJ, Eshleman VR. The neutral atmosphere of Venus as studied. with the Mariner $\mathrm{V}$ radio occultation experiments. Astron J 1971; 76: 123-40.

[16] Bech J, Sairouni A, Codina B, Lorente J, Bebbington D. Weather radar anaprop conditions at a Mediterranean coastal site. Physics and Chemistry of the Earth, Part B: Hydrology, Oceans and Atmosphere 2000; 25(10-12): 829-32.

[17] Lopez P. A 5-yr 40-km-resolution global climatology of superrefraction for ground-based weather radars. J Appl Meteorol Climatol 2009; 48(1): 89-110.

[18] Smith EK, Weintraub S. The constants in the equation for atmospheric refractive index at radio frequencies. Proc IRE 1953; 41:1035-37.

[19] Vespe F, Benedetto C, Pacione R. Water vapor retrieved by GNSS radio occultation t6echnique with no external information? Radio Occultation Science Workshop, 2002, Boulder, Colorado.

[20] Demuth H, Beale M, Hagan M. Neural network toolbox for use with Matlab, User's Guide v.6, The MathWorks, 2008.

[21] Smith WL, Woolf HM. The use of eigenvectors of statistical covariance matrices for interpreting satellite sounding radiometer observations. J Atmos Sci 1976; 33: 1127-40.

[22] Marquardt D. An algorithm for least square estimation on nonlinear parameters. J Soc Appl Math 1963; 431-441.

[23] Hagan M, Menhaj M. Training feed-forward networks with the Marquardt algorithm. IEEE Trans Neural Networks 1994; 5(6): 989-93.

[24] Born M, Wolf E. Principles of Optics. $6^{\text {th }}$ ed. Pergamon: New York, USA 1980.

[25] Hunt D, Schreiner B, Sokolovskiy S, Syndergaard S, Rocken C. COSMIC Data Analysis and Archive Center Status and Data Product Description. $2^{\text {nd }}$ GPS RO Data Users Workshop, Lansdowne VA, 2005 USA. 